\title{
MTHFR Gene C677T Polymorphism in Autism Spectrum Disorders
}

\author{
Elif Funda Sener, ${ }^{1}$ Didem Behice Oztop, ${ }^{2}$ and Yusuf Ozkul ${ }^{3}$ \\ ${ }^{1}$ Department of Medical Biology, Erciyes University Medical School, 38039 Kayseri, Turkey \\ ${ }^{2}$ Department of Child Psychiatry, Erciyes University Medical School, 38039 Kayseri, Turkey \\ ${ }^{3}$ Department of Medical Genetics, Erciyes University Medical School, 38039 Kayseri, Turkey
}

Correspondence should be addressed to Elif Funda Sener; efefunda@yahoo.com

Received 25 August 2014; Revised 22 October 2014; Accepted 23 October 2014; Published 6 November 2014

Academic Editor: Francine Durocher

Copyright (c) 2014 Elif Funda Sener et al. This is an open access article distributed under the Creative Commons Attribution License, which permits unrestricted use, distribution, and reproduction in any medium, provided the original work is properly cited.

\begin{abstract}
Aim. Autism is a subgroup of autism spectrum disorders, classified as a heterogeneous neurodevelopmental disorder and symptoms occur in the first three years of life. The etiology of autism is largely unknown, but it has been accepted that genetic and environmental factors may both be responsible for the disease. Recent studies have revealed that the genes involved in the folate/homocysteine pathway may be risk factors for autistic children. In particular, C677T polymorphism in the MTHFR gene as a possible risk factor for autism is still controversial. We aimed to investigate the possible effect of C677T polymorphism in a Turkish cohort. Methods. Autism patients were diagnosed by child psychiatrists according to DSM-IV and DSM-V criteria. A total of 98 children diagnosed as autistic and 70 age and sex-matched children who are nonautistic were tested for C677T polymorphism. This polymorphism was studied by using polymerase chain reaction-restriction fragment length polymorphism (PCR-RFLP) methods. Results. MTHFR 677T-allele frequency was found to be higher in autistic children compared with nonautistic children ( $29 \%$ versus $24 \%$ ), but it was not found statistically significant. Conclusions. We conclude that other MTHFR polymorphisms such as A1298C or other folate/homocysteine pathway genes may be studied to show their possible role in autism.
\end{abstract}

\section{Introduction}

Autism spectrum disorders (ASDs) include autistic disorder (or classic autism), Asperger syndrome, pervasive developmental disorders-not otherwise specified (PDD-NOS), and childhood disintegrative disorder. As a childhood disease, autism is characterized by three core symptoms with impaired reciprocal social interaction and communication, a pattern of repetitive behavior and/or restricted interests [14]. Clinical diagnosis of ASD is based on behavioral history and behavioral assessments of the clinical features by child psychiatrists. Clinicians usually use validated diagnostics tools. Much more attention has been given to the significant increase in the reported incidence of ASDs in the world today [5]. The rise of autism has been attributed to the changes in diagnostic tools, professional awareness of the disease, and broader diagnostic definition $[5,6]$. Today, the prevalence of the disease is reported to be approximately $1 / 80-100[4,5,7]$.
ASDs are accepted as multifactorial inheritance with $90 \%$ genetic background $[5,8]$. As a complex neurodevelopmental disorder, the phenotype and severity of autism are extremely heterogeneous with differences from one patient to another $[6,8,9]$. This heterogeneity involves both locus and allelic heterogeneity in ASD cases [10].

Despite the lack of success in identifying the candidate genes which are responsible for the majority of ASD cases, epigenetic modifications of genes important for normal brain development and growth and cognitive function and behavior are involved in the etiology of ASDs. The term epigenetics means reversible heritable changes in gene expression regulation by modulating rather than by changes in the nucleotide sequence of a gene [7]. Abnormal methylation patterns as epigenetic defects have been implicated in idiopathic ASDs $[11,12]$ as well as ASD-associated syndromes such as Rett's syndrome, Angelman syndrome, Prader-Willi syndrome, and Fragile-X syndrome $[12,13]$. 
In recent years, some studies have revealed that polymorphisms of the genes are involved in the folate/homocysteine pathway as risk factors for autistic children [14-17]. There is a great deal of evidence suggesting that DNA methylation defects are associated with ASDs, and the role of the methylenetetrahydrofolate reductase (MTHFR) gene in folate metabolism may contribute to epigenetic mechanisms that modify complex gene expression, thus causing autism. MTHFR is one of the most important enzymes in the folate pathway. It converts 5,10-methylenetetrahydrofolate to 5-methylenetetrahydrofolate and regulates the intracellular flow of folate. C677T polymorphism in the MTHFR gene (A222V, rs1801133) is associated with a decrease in enzymatic activity to $35-70 \%$ in homozygotes [18].

To date, there have been some case-control studies of MTHFR functional polymorphisms in autism and ASD [14, $15,17]$. Herein we aimed to present our findings on MTHFR C677T polymorphism in a total of 98 autistic patients in Turkey.

\section{Material and Methods}

2.1. Study Design. MTHFR is probably relevant in autism and we wanted to test our study group in Turkey. The study group was collected between May 2009 and May 2014. Patients were diagnosed by using the Diagnostic and Statistical Manual of Mental Disorder, Fourth Edition (DSMIV) and DSM-V $[19,20]$. Exclusion criteria included those who were under the age of 3 and/or diagnosis of genetic or neurological disorder associated with autism. All of the patients had a diagnosis of autism and were followed up at the Department of Child Psychiatry. The case group ( $n=$ 98) comprised patients with autism and the control group $(n=70)$ comprised healthy children. There were 81 simplex families and 8 multiplex families in the case group. The control group was randomly selected and matched for age and sex for this study. In the study group, there were 71 males and 27 females. Information spanning a minimum of three generations of family history was obtained and simplex families did not reveal further cases of ASDs. All of the cases met the clinical criteria for autism found in the absence of a known etiology with detectable chromosomal defects using the standard karyotyping procedure and other genetic syndromes, that is, Fragile-X syndrome, Rett's syndrome, and Angelman syndrome. The control group consisted of 46 males and 24 females. This study was approved by ethical committee in Erciyes University and informed consent was obtained from all the participants.

2.2. DNA Extraction and Genotyping. Two $\mathrm{mL}$ blood samples were obtained from patients with autism and from the control group. Genomic DNA was extracted according to the standard protocols of Roche (Roche Magna Pure LC, Germany). MTHFR C677T polymorphism (rs1801133) was examined by polymerase chain reaction- (PCR-)restriction fragment length polymorphism (RFLP). PCR fragments were amplified from $20 \mathrm{ng}$ of each DNA sample in $50 \mu \mathrm{L}$ PCR mix containing $0.5 \mathrm{U} / \mathrm{mL}$ Taq DNA polymerase, $1.5 \mathrm{mM} \mathrm{MgCl}_{2}$,

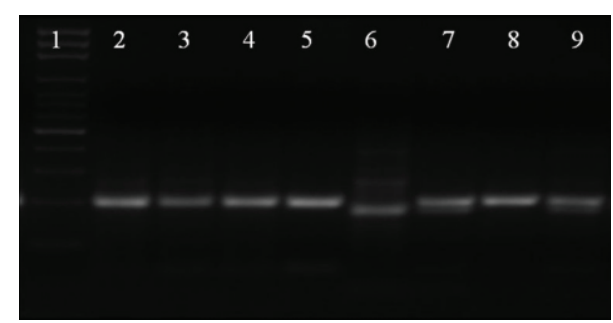

FIGURE 1: Image of agarose gel electrophoresis after Hinf 1 restriction enzyme digestion. 1: 100 base pair DNA ladder. 2: PCR product, 3-5, 8: CC genotype, 6: TT genotype, 7, 9: CT genotype.

1X concentration of the buffer, $2.5 \mathrm{mM}$ concentration of deoxynucleotide triphosphate (dNTP), and 10 pmol of each primer. Primer sequences are summarized in Table 1. PCR cycling conditions included initial denaturation at $94^{\circ} \mathrm{C}$ for $2 \mathrm{~min}$, followed by $40 \mathrm{cycles}$ of $30 \mathrm{sec}$ at $94^{\circ} \mathrm{C}, 30 \mathrm{sec}$ at $62^{\circ} \mathrm{C}$, and $30 \mathrm{sec}$ at $72^{\circ} \mathrm{C}$, and a final extension step at $72^{\circ} \mathrm{C}$ for $7 \mathrm{~min}$. One hundred and ninety-eight base pairs of PCR products were then digested overnight at $37^{\circ} \mathrm{C}$ with Hinf I restriction enzyme and checked with 3\% agarose gel electrophoresis [21]. Every PCR was accompanied with a negative control without any genomic DNA and a positive control with genomic DNA that is digested completely by the particular restriction enzyme (Figure 1).

2.3. Statistical Analysis. To assess the data normality, histogram and $q-q$ plots were examined; also Shapiro-Wilk's test was applied. To compare the differences between disease groups, Mann-Whitney $U$ test was used for continuous variables. Pearson's chi-square analysis was used to determine the relationship between genes in ASD. Odds ratios with 95\% confidence intervals were calculated for risk assessment. Analyses were conducted using R 3.1.0 (http://www.rproject.org/) software by considering a $P$ value less than 0.05 statistically significant.

\section{Results}

In the study group, there were 71 males $(72.4 \%)$ and 27 $(27.6 \%)$ females. The mean age of cases was $6.0(3.8-8.0)$. There were $46(65.7 \%)$ male controls and $24(34.3 \%)$ female controls. The mean age of controls was 5.0 (4.0-6.0). There was not any significant difference between the groups for this polymorphism $(P=0.237)$. The heterozygote genotype was associated with 1.3-fold (95\% CI: 0.70-2.41) risk for autism and this risk was not statistically significant (Table 2). However, MTHFR 677T-variant allele frequencies in cases and controls were $29 \%$ and $24 \%$, respectively. When we adjusted the analysis according to age effect, we again did not find any statistically significant difference between the groups $(P=0.176$; 95\% CI: 1.37 (0.73-2.58)).

\section{Discussion}

Autism is accepted to have a complex etiology involving both genetic and environmental factors with epigenetic 
TABLE 1: Primer sequences and genotype differences after Hinf I enzymatic digestion of MTHFR C677T polymorphism.

\begin{tabular}{|c|c|c|c|}
\hline Primer sequences & CC genotype & CT genotype & TT genotype \\
\hline $\begin{array}{l}\text { F: } 5^{\prime} \text {-TGAAGGAGAAGGTGTCTGCGGGA-3' } \\
\text { R: } 5^{\prime} \text {-AGGACGGTGCGGTGAGAGTG-3' }\end{array}$ & $198 \mathrm{bp}$ & $\begin{array}{l}198 \mathrm{bp} \\
175 \mathrm{bp} \\
23 \mathrm{bp}\end{array}$ & $\begin{array}{l}175 \mathrm{bp} \\
23 \mathrm{bp}\end{array}$ \\
\hline
\end{tabular}

F: forward, R: reverse.

TABLe 2: Genotype and allelic distribution of MTHFR C677T polymorphism in patients with autism and control groups.

\begin{tabular}{lcccc}
\hline & $\begin{array}{c}\text { Control } \\
(n=70)\end{array}$ & $\begin{array}{c}\text { Patients } \\
(n=98)\end{array}$ & $P$ value & $\begin{array}{c}\text { OR } \\
(95 \% \mathrm{CI})\end{array}$ \\
\hline Female & $24(34.3)$ & $27(27.6)$ & 0.349 & 1.00 \\
Male & $46(65.7)$ & $71(72.4)$ & & $1.37(0.71-2.66)$ \\
\hline $\begin{array}{l}\text { MTHFR } \\
\text { C677T }\end{array}$ & & & & \\
$\quad$ CC & $37(52.9)$ & $44(44.9)$ & 0.237 & 1.00 \\
CT & $33(47.1)$ & $51(52.0)$ & & $1.30(0.70-2.41)$ \\
TT & $0(0.0)$ & $3(3.1)$ & & - \\
\hline C allele & 0.76 & 0.71 & & \\
T allele & 0.24 & 0.29 & & \\
\hline
\end{tabular}

OR: odds ratio; CI: confidence interval.

modifications $[12,22,23]$. In this study, we investigated a key gene in folate metabolism. This gene and its polymorphisms have been previously reported to be associated with ASD susceptibility in some case-control studies to date $[7,15,17]$. We could not demonstrate a possible risk for MTHFR C677T polymorphism in autism cases.

Liu et al. selected two common polymorphisms (C677T and A1298C) to test simplex and multiplex ASD families because of the association of reduced MTHFR enzyme activity. The $\mathrm{T}$ allele was more prevalent in children with an ASD (42.9\%) compared to controls (32.3\%) and this finding differed significantly from those in the comparison group with a $P$ value of 0.0004 . However, the frequency of the heterozygous 677CT genotype in the ASD group (47.8\%) did not differ from that in the controls (43.2\%). The allele and genotype frequencies of the polymorphism in multiplex families were very similar to those in the control group. Finally, they suggested that reduced MTHFR activity is a risk factor for autism only in simplex families [7]. James et al. observed low methionine, low S-adenosyl methionine (SAM)/S-adenosyl homocysteine (SAH) ratio, low cysteine, and low glutathione levels in their study group. They detect significant alteration in homocysteine levels in autism [24]. Paşca et al. studied C677T polymorphism of the MTHFR gene in three groups of children diagnosed with autism $(n=15)$, Asperger syndrome $(n=5)$, and PDD-NOS $(n=19)$ and their age- and sex-matched controls $(n=25)$. The results showed a normal distribution of the C677T polymorphism in children with ASDs, but the frequency of the T allele was slightly more prevalent in autistic patients. They, therefore, thought that a possible role for the alterations in one carbon metabolism existed in the pathophysiology of ASDs [25].
In a Chinese Han population, the frequency of the TT genotype of MTHFR 677 was significantly higher in children with autism (16.1\%) than in controls (8.6\%). According to this finding, Guo et al. suggested that MTHFR C677T is a risk factor for autistic patients in their population [26]. One hundred and sixty-eight children with a confirmed diagnosis of autism or PDD were investigated by Boris et al. in 2004 . Their data demonstrated that $677 \mathrm{CT}$ polymorphism, whether in homozygous or heterozygous state, was significantly associated with ASD. They observed an increased frequency of the TT genotype in the autistic children (23\%) compared to $11 \%$ in the control population [14]. In another study, the case group comprised 151 patients with idiopathic ASDs and 100 healthy controls. The frequency of the $\mathrm{T}$ allele was the same $(0.38$ versus 0.35$)$ between the groups $(P=0.77)$. The genotype distribution did not reveal significant differences between cases and controls $(P=0.72)$ [27].

An interesting study was carried on about behavioral problems in children with autism. Goin-Kochel et al. hypothesized that autistic children who carry the homozygous genotype for the MTHFR 677TT would exhibit more behavioral problems and/or more severe problematic behaviors than homozygous wild-type (CC) individuals. Their results did not suggest such a relationship between genotypes for MTHFR 677CT and developmental regression. They also showed that four behaviors (current complex body movements, direct gaze, a history of self-injurious behavior, and current overactivity) were more common and problematic among those with a heterozygous state as compared to homozygous wildtype individuals [16]. For Park et al. MTHFR 677CT and 1298AC polymorphisms were tested in Korean population. 677CT/1298AC was significantly associated with 2.11-fold increased risk of ASD compared with the combination of 677CC/1298AA genotypes [28]. A meta-analysis was performed by Pu et al. in 2013. They investigated the relationship of the MTHFR polymorphisms (C677T and A1298C) and the risk of ASD. Eight case-control studies (1672 patients with ASD and 6760 controls) were included for this metaanalysis. Their results show an association of MTHFR C677T polymorphism with increased susceptibility to ASD [29].

In our study genotyping for MTHFR 677CT revealed 44 (44.9\%) children with the CC genotype, 51 (52\%) children with the CT genotype, and 3 (3.1\%) children with the TT genotype in cases. We did not detect any homozygous polymorphism in the control group and this result was not significantly different between the groups. T allele frequency was higher in the patients. Our results were in accordance with dos Santos et al.s study. Thus, contrary to some studies we could not demonstrate an association between this polymorphism and autism. One limitation of this study is 
the small sample size of autism families. Differences due to the genetic heterogeneity of autism, ethnic variation, recruiting strategies, family types (simplex or multiplex), sample size, and/or other factors that we could account might give these results.

\section{Conclusion}

We suggest replicating the study with larger well-characterized simplex families with nonautistic siblings, to determine the presence or absence of common MTHFR SNPs (especially $\mathrm{C} 677 \mathrm{~T}$ and $\mathrm{A} 1298 \mathrm{C}$ ). We recommend investigating this risk in a larger sample size with the same diagnostic criteria, same ethnicity and paying great attention to gender distribution to get reliable results in ASD.

Other environmental risk factors should be considered; in particular, the methylation/transsulfation and/or catecholamine-O-methyltransferase (COMT) pathways should be investigated in future studies.

\section{Conflict of Interests}

The authors declare that they have no conflict of interests.

\section{Acknowledgment}

The authors declare that this study has no supporting grant.

\section{References}

[1] A. M. Persico and V. Napolioni, "Autism genetics," Behavioural Brain Research, vol. 251, pp. 95-112, 2013.

[2] J. H. Miles, "Autism spectrum disorders-a genetics review," Genetics in Medicine, vol. 13, no. 4, pp. 278-294, 2011.

[3] B. Devlin and S. W. Scherer, "Genetic architecture in autism spectrum disorder," Current Opinion in Genetics \& Development, vol. 22, no. 3, pp. 229-237, 2012.

[4] P. Szatmari, X.-Q. Liu, J. Goldberg et al., "Sex differences in repetitive stereotyped behaviors in autism: implications for genetic liability," American Journal of Medical Genetics Part B: Neuropsychiatric Genetics, vol. 159, no. 1, pp. 5-12, 2012.

[5] N. J. Mendelsohn and G. B. Schaefer, "Genetic evaluation of autism," Seminars in Pediatric Neurology, vol. 15, no. 1, pp. 27-31, 2008.

[6] G. B. Schaefer and N. J. Mendelsohn, "Clinical genetics evaluation in identifying the etiology of autism spectrum disorders," Genetics in Medicine, vol. 10, no. 4, pp. 301-305, 2008.

[7] X. Liu, F. Solehdin, I. L. Cohen et al., "Population- and familybased studies associate the MTHFR gene with idiopathic autism in simplex families," Journal of Autism and Developmental Disorders, vol. 41, no. 7, pp. 938-944, 2011.

[8] V. Eapen, "Genetic basis of autism: is there a way forward?" Current Opinion in Psychiatry, vol. 24, no. 3, pp. 226-236, 2011.

[9] J. L. Silverman, M. Yang, C. Lord, and J. N. Crawley, "Behavioural phenotyping assays for mouse models of autism," Nature Reviews Neuroscience, vol. 11, no. 7, pp. 490-502, 2010.

[10] P. Chaste and M. Leboyer, "Autism risk factors: genes, environment, and gene-environment interactions," Dialogues in Clinical Neuroscience, vol. 14, no. 3, pp. 281-292, 2012.
[11] Y.-H. Jiang, T. Sahoo, R. C. Michaelis et al., "A mixed epigenetic/genetic model for oligogenic inheritance of autism with a limited role for UBE3A," American Journal of Medical Genetics Part A, vol. 131, no. 1, pp. 1-10, 2004.

[12] N. C. Schanen, "Epigenetics of autism spectrum disorders," Human Molecular Genetics, vol. 15, no. 2, pp. R138-R150, 2006.

[13] R. P. Ghosh, R. A. Horowitz-Scherer, T. Nikitina, L. M. Gierasch, and C. L. Woodcock, "Rett syndrome-causing mutations in human MeCP2 result in diverse structural changes that impact folding and DNA interactions," The Journal of Biological Chemistry, vol. 283, no. 29, pp. 20523-20534, 2008.

[14] M. Boris, A. Goldblatt, J. Galanko, and J. James, "Association of MTHFR gene variants with autism," Journal of the American Physician and Surgeons, vol. 9, no. 4, pp. 106-108, 2004.

[15] S. J. James, S. Melnyk, S. Jernigan et al., "Metabolic endophenotype and related genotypes are associated with oxidative stress in children with autism," American Journal of Medical Genetics Part B: Neuropsychiatric Genetics, vol. 141, no. 8, pp. 947-956, 2006.

[16] R. P. Goin-Kochel, A. E. Porter, S. U. Peters, M. Shinawi, T. Sahoo, and A. L. Beaudet, "The MTHFR $677 C \rightarrow$ T polymorphism and behaviors in children with autism: exploratory genotype-phenotype correlations," Autism Research, vol. 2, no. 2, pp. 98-108, 2009.

[17] N. S. Mohammad, J. M. N. Jain, K. P. Chintakindi, R. P. Singh, U. Naik, and R. R. D. Akella, "Aberrations in folate metabolic pathway and altered susceptibility to autism," Psychiatric Genetics, vol. 19, no. 4, pp. 171-176, 2009.

[18] P. Frosst, H. J. Blom, R. Milos et al., "A candidate genetic risk factor for vascular disease: a common mutation in methylenetetrahydrofolate reductase," Nature Genetics, vol. 10, no. 1, pp. 111113, 1995.

[19] American Psychiatric Association, Diagnostic and Statistical Manual of Mental Disorders (DSM-IV), American Psychiatric Press, Washington, DC, USA, 4th edition, 2000.

[20] American Psychiatric Association, Diagnostic and Statistical Manual of Mental Disorders, American Psychiatric Press, Washington, DC, USA, 5th edition, 2013.

[21] M. Ozkan, S. Sivgin, I. Kocyigit et al., "Do thrombophilic gene mutations have a role on thromboembolic events in cancer patients?" Asia-Pacific Journal of Clinical Oncology, vol. 8, no. 3, pp. e34-e41, 2012.

[22] T. Kubota, K. Miyake, and T. Hirasawa, "Epigenetic understanding of gene-environment interactions in psychiatric disorders: a new concept of clinical genetics," Clinical Epigenetics, vol. 4, no. 1, article 1, 2012.

[23] M. J. Millan, "An epigenetic framework for neurodevelopmental disorders: from pathogenesis to potential therapy," Neuropharmacology, vol. 68, pp. 2-82, 2013.

[24] S. J. James, P. Cutler, S. Melnyk et al., "Metabolic biomarkers of increased oxidative stress and impaired methylation capacity in children with autism," The American Journal of Clinical Nutrition, vol. 80, no. 6, pp. 1611-1617, 2004.

[25] S. P. Paşca, E. Dronca, T. Kaucsár et al., "One carbon metabolism disturbances and the C677T MTHFR gene polymorphism in children with autism spectrum disorders," Journal of Cellular and Molecular Medicine, vol. 13, no. 10, pp. 4229-4238, 2009.

[26] T. Guo, H. Chen, B. Liu, W. Ji, and C. Yang, "Methylenetetrahydrofolate reductase polymorphisms C677T and risk of autism in the Chinese han population," Genetic Testing and Molecular Biomarkers, vol. 16, no. 8, pp. 968-973, 2012. 
[27] P. A. dos Santos, D. Longo, A. P. C. Brandalize, and L. Schüler-Faccini, "MTHFR C677T is not a risk factor for autism spectrum disorders in South Brazil," Psychiatric Genetics, vol. 20, no. 4, pp. 187-189, 2010.

[28] J. Park, M. Ro, J.-A. Pyun et al., "MTHFR 1298A>C is a risk factor for autism spectrum disorder in the Korean population," Psychiatry Research, vol. 215, no. 1, pp. 258-259, 2014.

[29] D. Pu, Y. Shen, and J. Wu, "Association between mthfr gene polymorphisms and the risk of autism spectrum disorders: a meta-analysis," Autism Research, vol. 6, no. 5, pp. 384-392, 2013. 

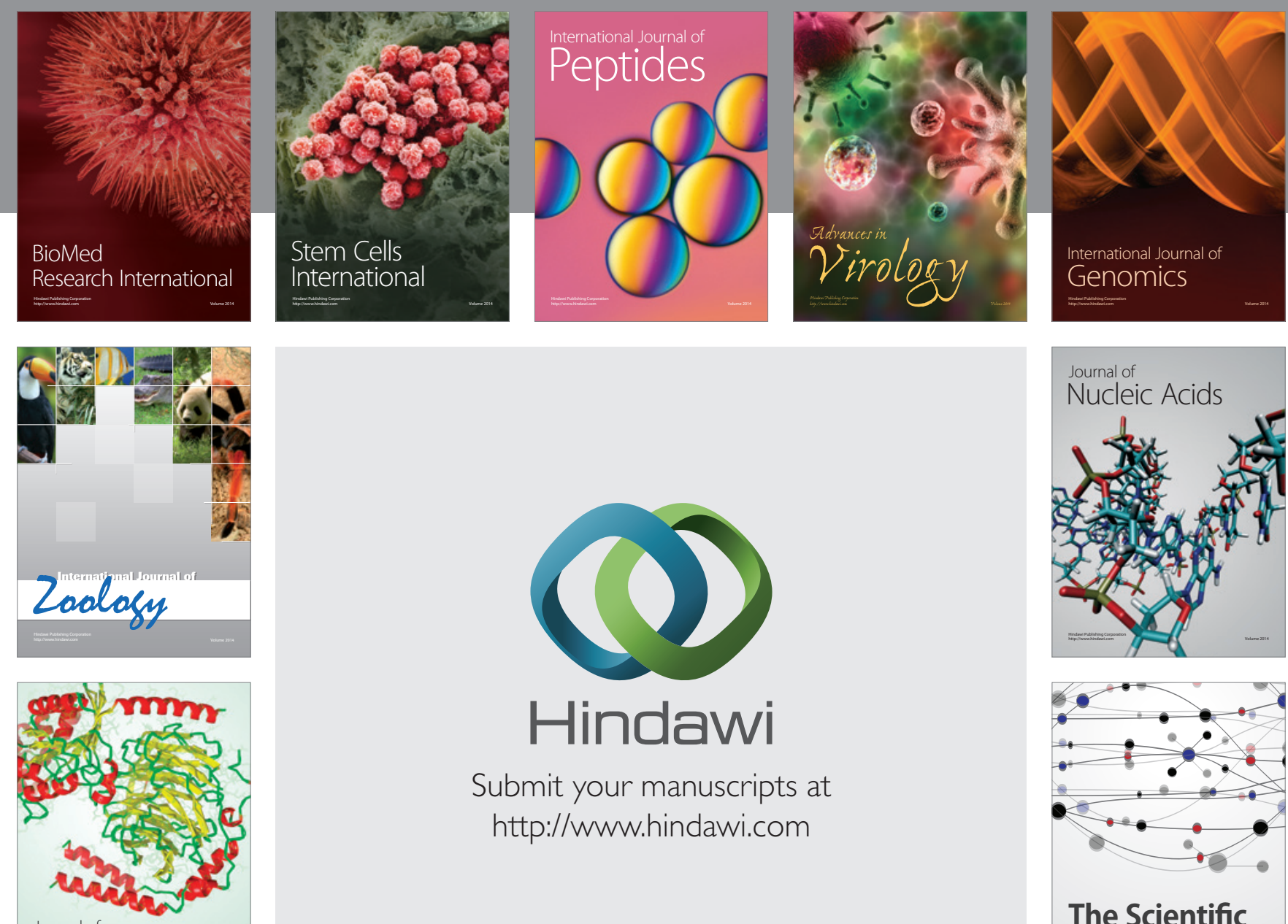

Submit your manuscripts at

http://www.hindawi.com

Journal of
Signal Transduction
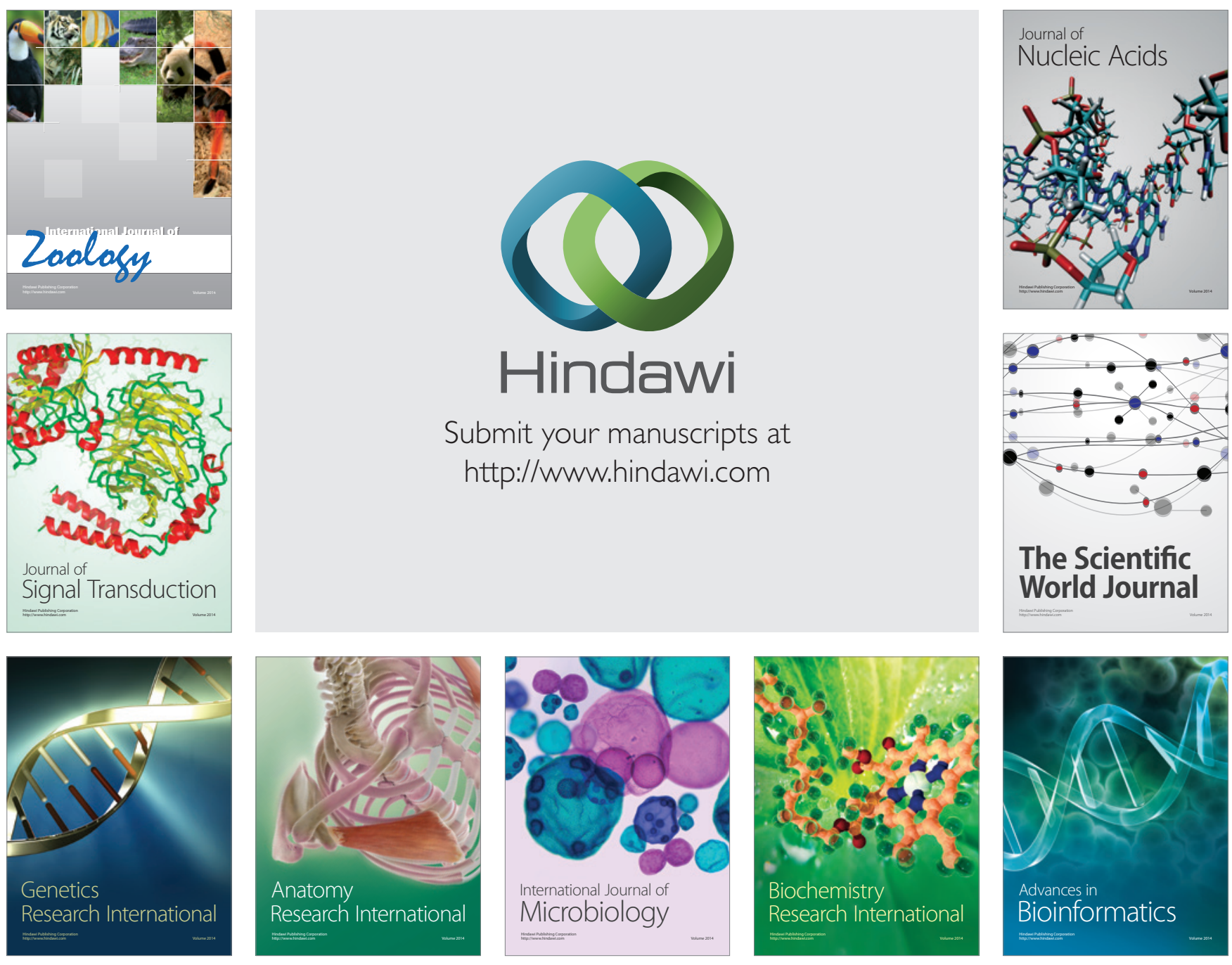

The Scientific World Journal
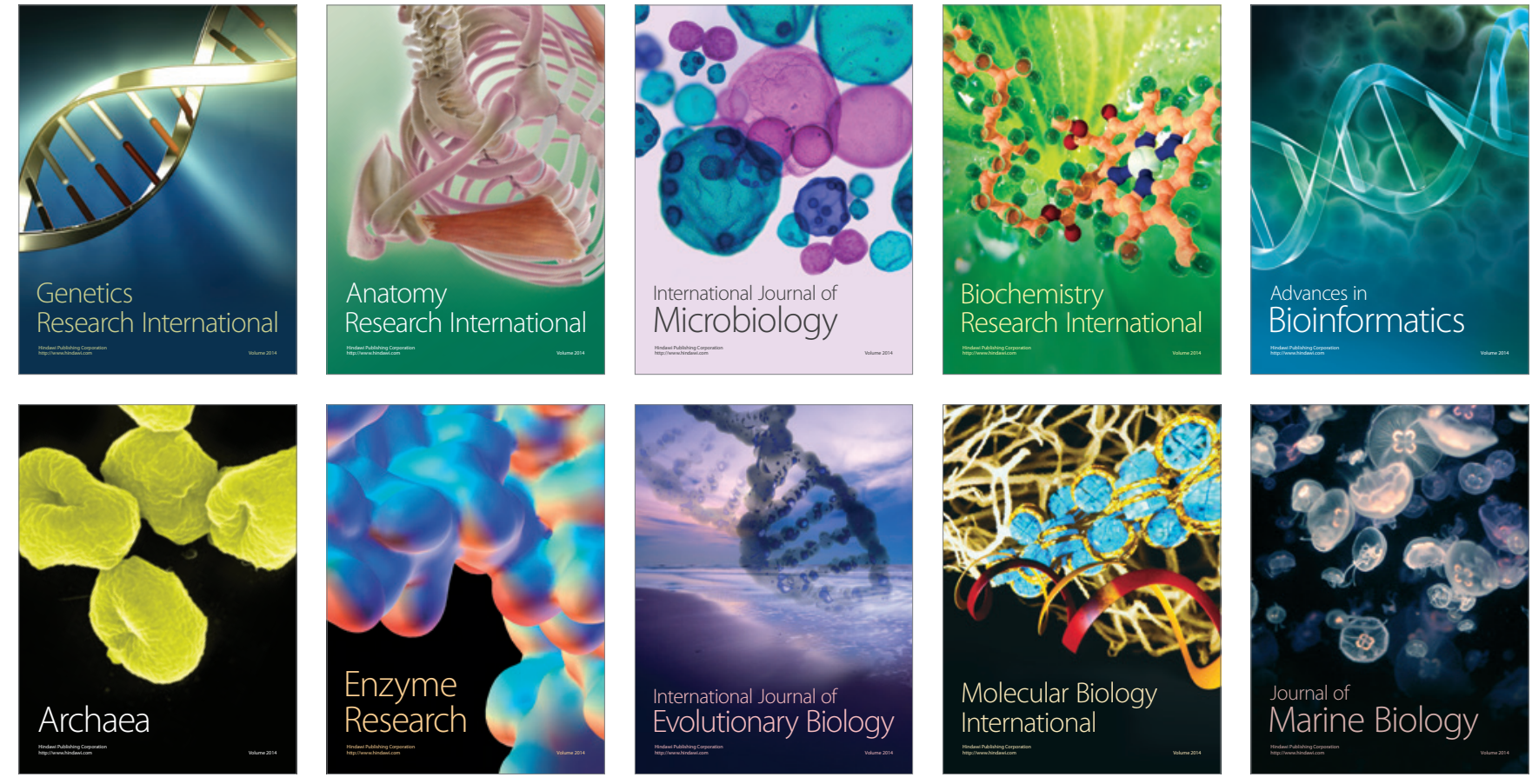